\title{
Preparation, Characterization and Applica- tion of Organic Conducting Polymer-Silica Nanocomposite Particles
}

\author{
Taito Sugiura $^{* 1, a)}$, Isao Komatsu ${ }^{* 2}$ \\ and Shuichi Maeda ${ }^{* 1,2}$ \\ ${ }^{* 1}$ Course of Electro Photo Optics, Graduate School of Engineering, \\ Tokai University \\ ${ }^{* 2}$ Course of Science and Technology, School of Science and Technology, \\ Tokai University
}

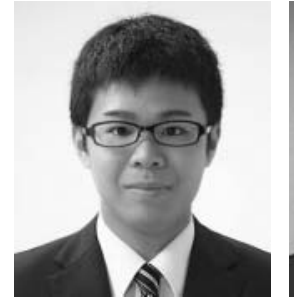

Taito Sugiura

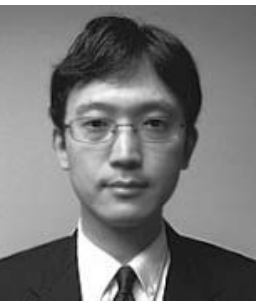

Isao Komatsu

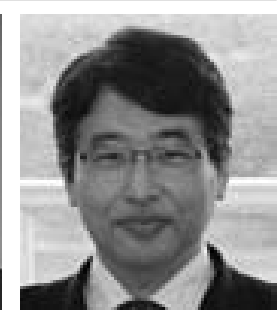

Shuichi Maeda

\begin{abstract}
We have prepared organic conducting nanocomposite particles which utilize polypyrrole/polyaniline as conducting parts and small silica particles as dispersants. The nanocomposite particles of polypyrrole/polyaniline and silica represent a potentially useful processable form of polypyrrole/polyaniline, a normally intractable conducting polymer. Transmission electron microscopy (TEM) studies confirm that the polypyrrole-silica/poly aniline-silica nanocomposite particles are made up of microaggregates of the original small silica particles, which give rise to a raspberry morphology. The particle diameter of the nanocomposites as measured by the TEM observation can be varied over the range $200-400 \mathrm{~nm}$ depending on the colloid synthesis conditions. We demonstrated that the polypyrrole-silica/polyaniline-silica nanocomposite particles can be utilized as display elements for electrophoretic display due to their high colloid stabilities. The electrical conductivities of polypyrrole-silica/ polyaniline-silica nanocomposite particles are measured and the paper sheets coated with these nanocomposite particles have higher conductivities compared to a conventional paper sheet. The polypyrrole-silica/polyanilinesilica nanocomposite particles can be utilized as conductive paper sheets.
\end{abstract}

Keywords : Conductive Sheets, Polyaniline, Polypyrrole, Colloidal silica, Printed electronics, Display elements, Conductive paper

\section{Introduction}

There has been increasing interest in conducting inks ${ }^{11}$

${ }^{* 1} 4-1-1$ Kitakaname, Hiratsuka-shi, Kanagawa 259-1292, Japan

${ }^{\text {a) }}$ E-mail : 4bahm004@mail.tokai-u.jp

Received : June 1, 2015 Accepted : September 9, 2015

J-STAGE Advance Published : October 28, 2015 for the direct printing of conductive patterns using inkjet systems in the field of printed electronics. In general these conducting inks are made from metal nanoparticles such as silver ${ }^{2)}$ and copper $^{3)}$. On the other hand, recently, there has been increasing expectation for utilizing conducting inks in the field of biological applications. When considering the application of conducting inks in biological fields, organic inks are preferable rather than metallic inks. Therefore, we have started to investigate the organic ma- 


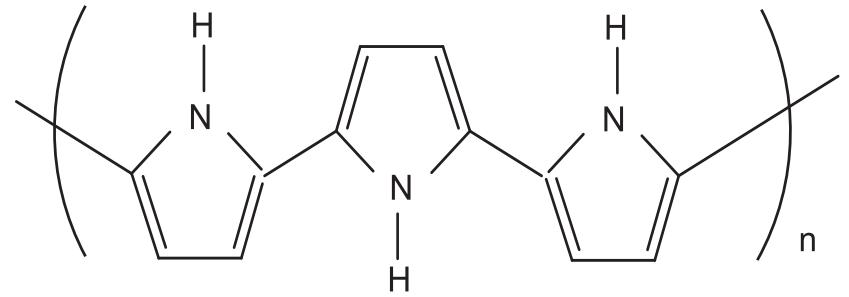

Fig. 1 Chemical structure of polypyrrole<smiles>CC(C)Nc1ccc(Nc2ccc(N=C3C=CC(=NC(C)(C)C)C=C3)cc2)cc1</smiles>

Fig. 2 Chemical structure of polyaniline

terials which play roles as conducting inks. One of the answers is to utilize organic conducting polymers.

Organic conducting polymers are usually insoluble and infusible due to their stiff structure, conjugated backbones $^{4)}$. These poor processability must be a major problem which has prevented these materials from acting as solutions. A useful approach to overcome the lack of processability of conducting polymers and to make them act as particles are to prepare colloidal dispersions of conducting polymers which are described in our previous work ${ }^{5)}$.

In this work, we use polypyrrole (Fig. 1) and polyaniline (Fig. 2) as conducting polymer because they are most attractive conducting polymers due to its good environmental stability ${ }^{6}$. We report the preparation of nanocomposite particles of polymers which give a rise to a raspberry morphology using small silica particles as a particulate dispersant in the field of electronic papers.

In the field of electronic paper, microcapsule electrophoretic display is most preferable system because it has good readability which is close to conventional paper. Particles as display elements of an electrophoretic display are usually covered with polymer surfactants which prevent these particles from $\mathrm{co}^{-}$aggregation. But it generally takes long time and high cost to find such surfactants. One of our present work, we utilize polymer-silica nanocomposite particles as display elements for electrophoretic display.

On the other hand, the results of the electrical conductivities of polymer-silica nanocomposite particles are compared with conventional photo paper sheet prepared under the same condition.

\section{Experiments}

2.1 Preparation of the polypyrrole-silica nanocomposite particles

The polypyrrole-silica nanocomposite particles which

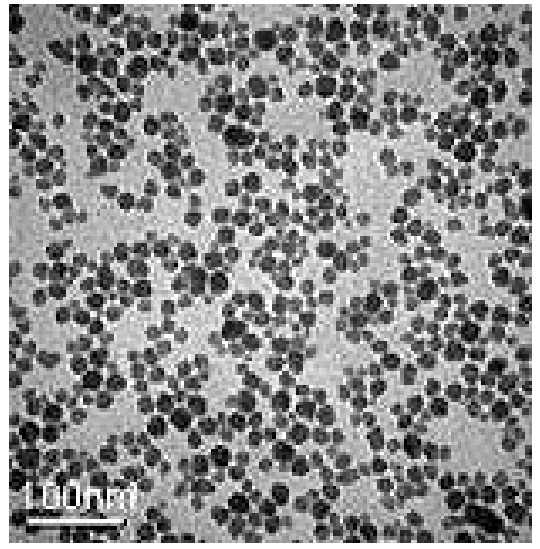

Fig. 3 Transmission electron micrograph of the original small silica particles (Nissan Chemical Products ; 10-15 $\mathrm{nm}$ particle diameter $)^{7)}$

act as conducting polymer were prepared as follows: 0.5-2. $0 \mathrm{~g}$ (dry weight) silica particles (Nissan Chemicals ; Snowtex-XS, 4-6 nm particle diameter; provided as 20 $\mathrm{w} / \mathrm{v} \%$ aqueous dispersion) were added to a solution of $\mathrm{FeCl}_{3}(4.55 \mathrm{~g})$ in de-ionized water (total solvent volume $=50 \mathrm{ml}$ ) at $25^{\circ} \mathrm{C}$ with constant stirring. A transmission electron micrograph of diluted dispersion of the Nissan silica particle samples ${ }^{7)}$ containing sodium are shown in Fig. 3. Pyrrole $(0.50 \mathrm{ml})$ was then injected via syringe into this stirred solution and the solution turned black within 1 minite (Fig.4). The polymerization was allowed to proceed for 2 hours. This reaction mixture was then centrifuged at 9,000 r. p. m. for 10 minites using a KURABO FB-8000 instrument and the resulting black sediment was redispersed in de-ionized water using an ultrasonic bath. This centrifugation-redispersion cycle was repeated three times in order to completely remove the excess small silica particles and (in) organic by-products from the larger polypyrrole-silica nanocomposite particles.

2.2 Preparation of the polyaniline-silica nanocomposite particles

The polyaniline-silica nanocomposite particles which act as conducting polymer were prepared as follows: 0.5-2. $0 \mathrm{~g}$ (dry weight) silica particles (Nissan Chemicals ; Snowtex-XS, 4-6 nm particle diameter; provided as 20 $\mathrm{w} / \mathrm{v} \%$ aqueous dispersion) were added to a solution of $\left(\mathrm{NH}_{4}\right)_{2} \mathrm{~S}_{2} \mathrm{O}_{8}(1.92 \mathrm{~g})$ in de-ionized water (total solvent volume $=50 \mathrm{ml})$ at $25^{\circ} \mathrm{C}$ with constant stirring. Aniline $(0.50$ $\mathrm{ml}$ ) was then injected via syringe into this stirred solution and the solution turned dark green within 3 minites (Fig. 5). The polymerization was allowed to proceed for 2 hours. This reaction mixture was then centrifuged at 9, 000 r. p. m. for 10 minites. using a KURABO FB-8000 instrument and the resulting dark green sediment was redispersed in de-ionized water using an ultrasonic bath. This centrifugation-redispersion cycle was repeated three 


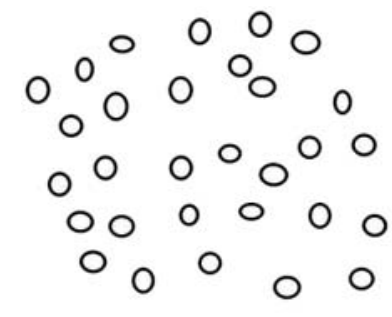

Silica particles

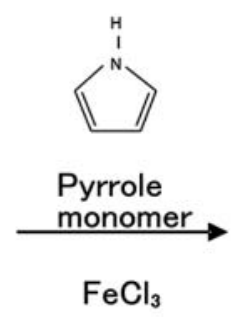

$\mathrm{FeCl}_{3}$

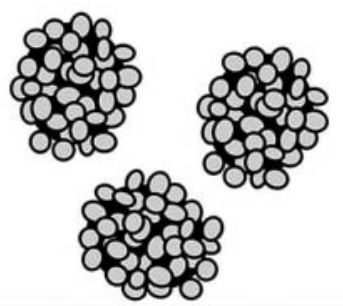

Polypyrrole-silica nanocomposite particles

Fig. 4 Schematic diagram showing formation of polypyrrole-silica nanocomposite particles from the original small silica particles which diameters are in $4-6 \mathrm{~nm}$

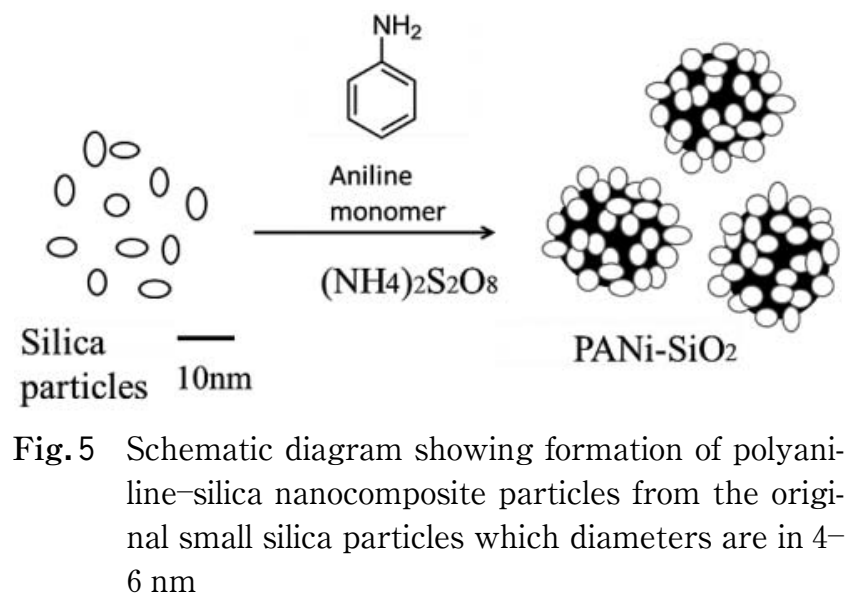

times in order to completely remove the excess small silica particles and (in) organic by-products from the larger polyaniline-silica nanocomposite particles.

\subsection{Characterization of the polymer-silica nanocom- posite particles}

Transmission electron microscopy (TEM) studies were made on dilute polypyrrole-silica nanocomposite particles and polyaniline-silica nanocomposite particles dried down on carbon-coated copper grids (Nisshin EM Co., Ltd. ; 200 mesh $\mathrm{Cu}$ ) using a HF-2200 (HITACHI., Ltd.) instrument at an operating voltage of $200 \mathrm{kV}$.

2.4 Development as display elements of electrophoretic displays

Procedures in order to use the polypyrrole-silica nanocomposite particles and polyaniline-silica nanocomposite particles as display elements of electrophoretic displays were carried out using the model experiment system described in Fig. 6. The polymer-silica nanocomposite particles were dispersed between two electrodes and floated at the boundary between two dielectric liquids with different specific gravities (Exon Chemical ; Isoper-G : specific gravity 0.75 and $3 \mathrm{M}$ Japan Limited ; $\mathrm{PF}-5080$ : specific gravity 1.70$)$. A D. C. voltage was set between the pair of electrodes and the motion of the polymer-silica nanocomposite particles were observed and recorded using a digi-

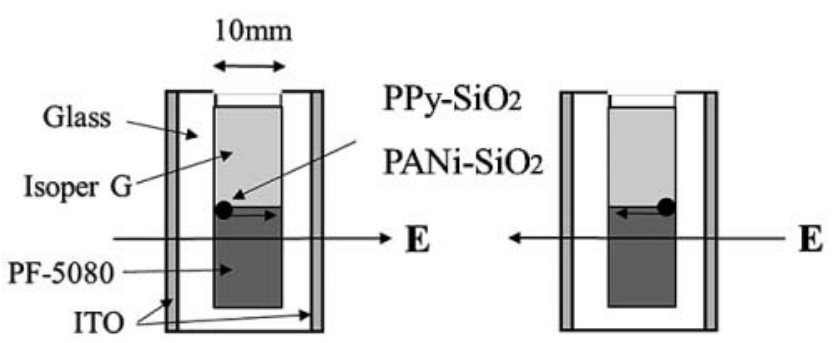

Fig. 6 Experimental apparatus for investigating the motion of the polypyrrole-silica nanocomposite particles and polyaniline-silica nanocomposite particles as display elements for electrophoretic display

tal microscope (KEYENCE VW-6000).

2.5 Preparation and characterization of the electrical conductivities of polypyrrole-silica nanocomposite paper sheet and polyaniline-silica nanocomposite paper sheet

Polypyrrole-silica nanocomposite particles and polyaniline-silica nanocomposite particles were coated on conventional photo sheet (KOKUYO ; KJ-G 14 A 4-50) using Auto File Applicator (TESTER SANGYO., Ltd.). The coated paper sheets (Fig. 7) were cut $10 \mathrm{~cm}$ on four sides and surface electrical resistance of the polymer-silica nanocomposite particles paper sheets were measured using ADVANTEST R 8340 Ultra High Resistance Meter and ADVANTEST R 12704 A Resistivity Chamber.

\section{Results and discussions}

3.1 Colloid stability of polypyrrole-silica nanocomposite particles

Summaries of the experimental data on the synthesis of polypyrrole-silica nanocomposite particles when using the Nissan Chemicals silica particles is presented in Table 1. The initial silica concentration (at least $1.0 \mathrm{w} / \mathrm{v} \%$ ) is required for the quantitative formation of stable polypyrrole -silica nanocomposite particles.

The colloid stability of one of the polypyrrole-silica nanocomposite particles (sample No.3) are compared with that of the bulk polypyrrole (sample No.1) as shown 


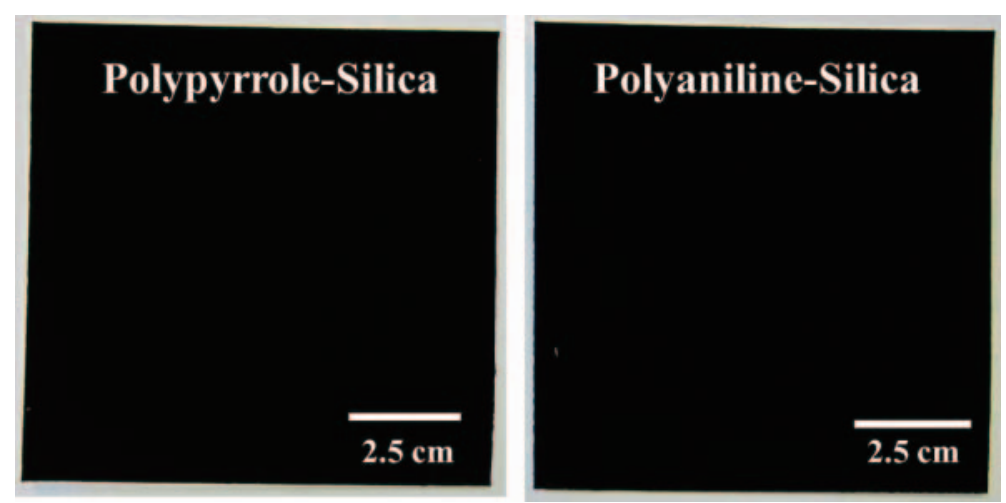

Fig. 7 Coated polypyrrole--silica nanocomposite particles paper sheet and coated polyaniline--silica nanocomposite particles paper sheet

Table 1 Effect of initial silica concentration on the particle formation of the polypyrrole-silica nanocomposites particles

\begin{tabular}{c|c|c}
\hline $\begin{array}{c}\text { Sample } \\
\text { No. }\end{array}$ & $\begin{array}{c}\text { Initial silica } \\
\text { concentration } \\
(\mathrm{w} / \mathrm{v} \%)\end{array}$ & $\begin{array}{c}\text { Colloid } \\
\text { formation }\end{array}$ \\
\hline 1 & 0.0 & NG \\
\hline 2 & 0.5 & Partially \\
\hline 3 & 1.0 & Yes \\
\hline 4 & 1.5 & Yes \\
\hline 5 & 2.0 & Yes \\
\hline
\end{tabular}

in Fig. 8. The long-term colloid stability of polypyrrole-silica nanocomposite particles relative to the bulk polypyrrole was confirmed in the observation. The polarity of the surface charge potential of the polypyrrole-silica nanocomposite particles related to the surface charge potential of original colloidal silica. Generally, isoelectric point of colloidal silica is around $\mathrm{pH} 2.0$, the silica colloid that we utilized is dispersed in $\mathrm{pH} 9.0 \sim 10.5$ solution. Therefore, the polarity of the surface charge potential of silica colloid can be said to be negative. Thus, the polypyrrole-silica nanocomposite particles are also negatively charged.

3.2 Colloid stability of polyaniline-silica nanocomposite particles

Summaries of the experimental data on the synthesis of polyaniline-silica nanocomposite particles when using the Nissan Chemicals silica particles is presented in Table 2. The initial silica concentration (at least $1.5 \mathrm{w} / \mathrm{v} \%$ ) is required for the quantitative formation of stable polyanilinesilica nanocomposite particles.

The colloid stability of one of the polyaniline--silica nanocomposite particles (sample No.9) are compared with that of the bulk polyaniline (sample No. 6) shown in Fig. 9. The long-term colloid stability of polyaniline--silica nanocom-

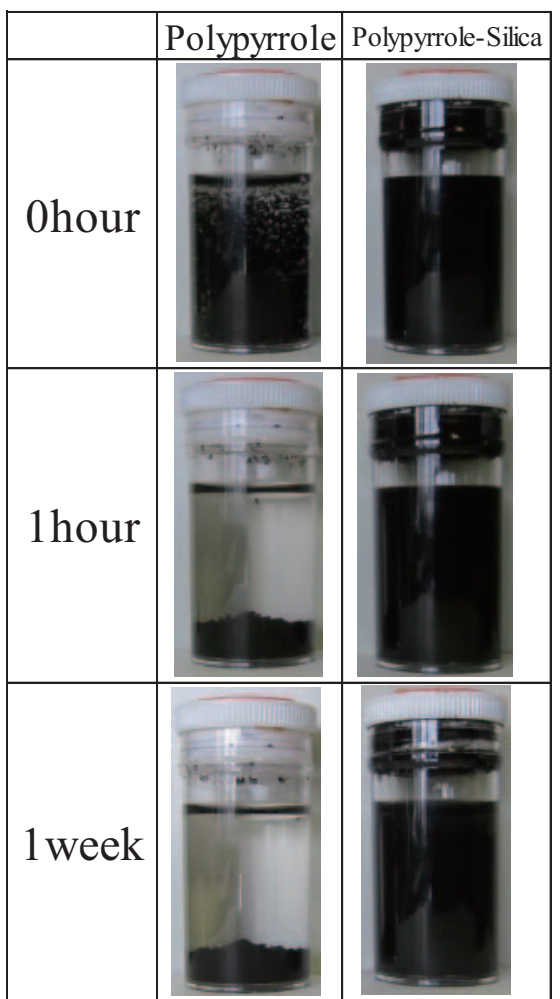

Fig. 8 Comparison of the colloid stabilities of the bulk polypyrrole (sample No.1) and the polypyrrolesilica nanocomposite particles (sample No. 3)

posite particles relative to the bulk polyaniline was confirmed in the observation.

\section{3 Morphology of the nanocomposite particles}

Typical transmission electron micrographs and scanning electron micrograph of diluted, dried polypyrrole-silica nanocomposite particles and polyaniline-silica nanocomposite particles are shown in Figs. 10, 11 and 12. These observations indicate that the schematic diagram of the formation of the polymer-silica nanocomposite particles from the original small silica particles described in Figs. 4 and 5 .

There are the two principal methods ${ }^{8)}$ by which particle 
Table 2 Effect of initial silica concentration on the particle formation of the polyaniline-silica nanocomposites particles

\begin{tabular}{c|c|c}
\hline $\begin{array}{c}\text { Sample } \\
\text { No. }\end{array}$ & $\begin{array}{c}\text { Initial silica } \\
\text { concentration } \\
(\mathrm{w} / \mathrm{v} \%)\end{array}$ & $\begin{array}{c}\text { Colloid } \\
\text { formation }\end{array}$ \\
\hline 6 & 0.0 & $\mathrm{NG}$ \\
\hline 7 & 0.5 & $\mathrm{NG}$ \\
\hline 8 & 1.0 & Partially \\
\hline 9 & 1.5 & Yes \\
\hline 10 & 2.0 & Yes \\
\hline
\end{tabular}

\begin{tabular}{|l|l|l|}
\hline & Polyaniline & Polyaniline-Silica \\
\hline 0hour & & \\
\hline 1 & & \\
\hline 1 hour & & \\
\hline
\end{tabular}

Fig. 9 Comparison of the colloid stabilities of the bulk polyaniline (sample No.6) and the polyaniline-silica nanocomposite particles (sample No.9)

aggregation can be prevented are steric stabilization and charge stabilization. In general the colloidal stability of silica sols comes from the latter method. In charge stabilization each colloid particle carries a positive or negative surface charge and the corresponding diffuse layer of ions of opposite charge extending into bulk solution. It is the interaction of these diffuse layers that is energetically unfavorable and leads to the mutual repulsion of two approaching particles.

The scanning electron micrograph depicted in Figs. 13 and 14 are of sample No. 1 and No. 6, polypyrrole and poly-

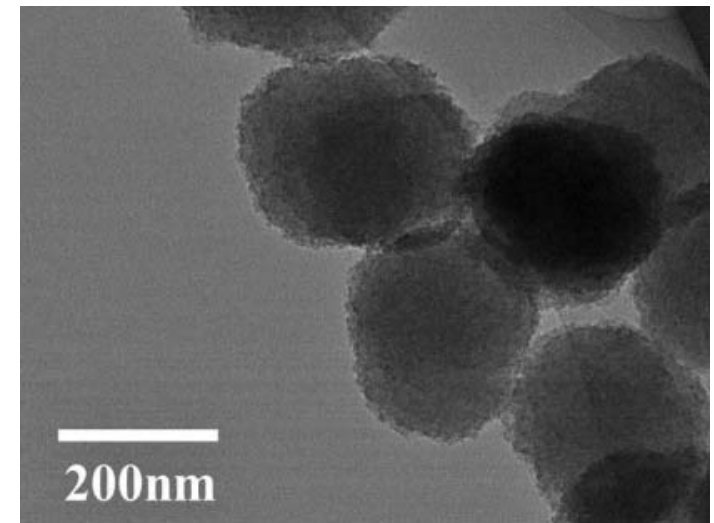

Fig. 10 Transmission electron micrograph of polypyrrole-silica nanocomposite particles

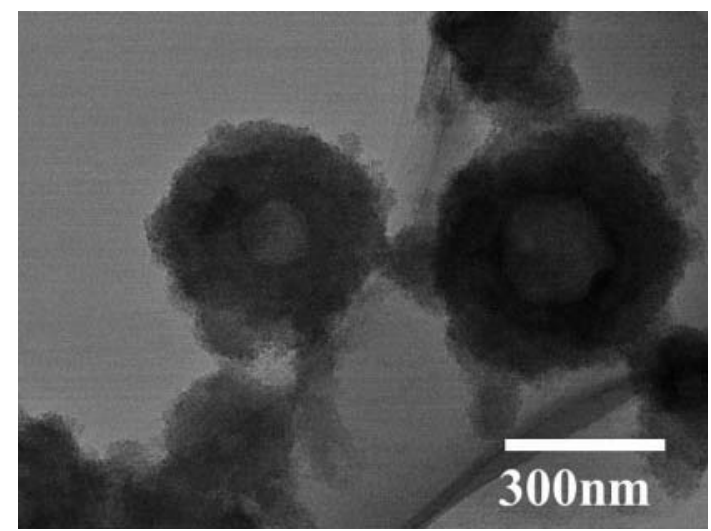

Fig. 11 Transmission electron micrograph of polyaniline-silica nanocomposite particles

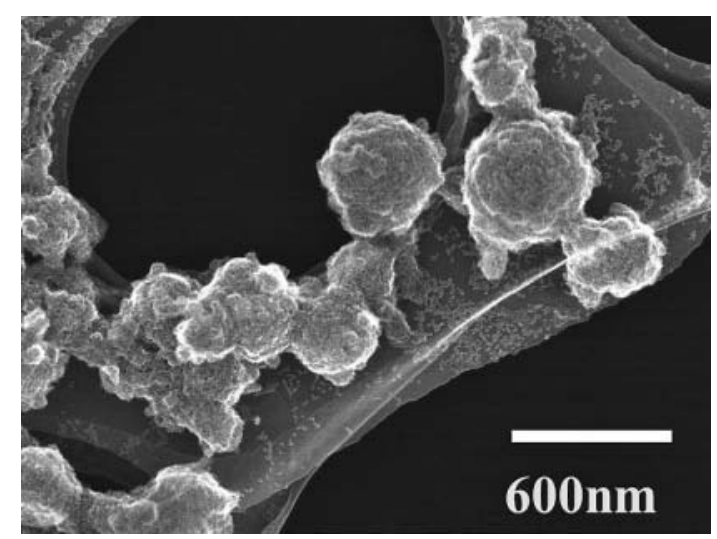

Fig. 12 Scanning electron micrograph of polyaniline-silica nanocomposite particles

aniline bulk powder precipitates in the absence of silica particles but under otherwise identical conditions to those used for polymer-silica nanocomposite particles synthesis. Clearly these particulates are larger than the dimensions of the polymer-silica nanocomposite particles prepared using the same chemical oxidant. Thus there is no doubt that the polymer morphologies within polymer-silica nanocomposite particles must be substantially different to that usually observed for conventional chemically synthesized 


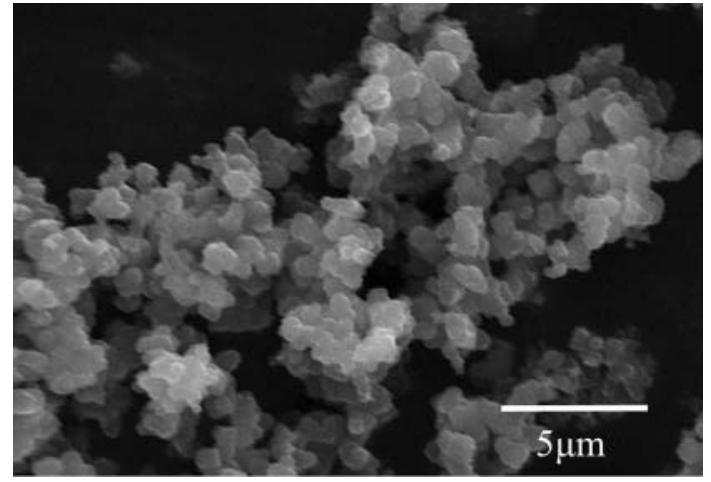

Fig.13 Scanning electron micrograph of bulk polypyrrole powder synthesized in the absence of silica sols (sample No.1)

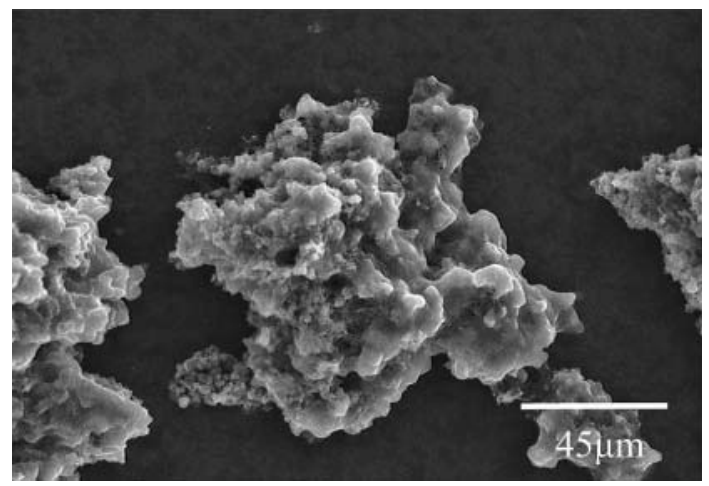

Fig. 14 Scanning electron micrograph of bulk polyaniline powder synthesized in the absence of silica sols (sample No.6)

polymer bulk powders.

3.4 As display elements for electrophoretic display

A summary of the experimental data of the motion of polypyrrole-silica nanocomposite particles and polyaniline -silica nanocomposite particles are presented in Table 3. When applying the electric voltage of $6,000 \mathrm{~V}$, the polymer-silica nanocomposite particles can be straightly moved from one of the electrodes to the other. Probably, there is a threshold between 5,000 and 6,000 V. Convert-

Table 3 Motion and speed of polymer-silica nanocomposite particles

\begin{tabular}{c|c|c}
\hline Voltage (V) & $\begin{array}{c}\text { Velocity of } \\
\text { Polypyrrole-Silica } \\
(\mathrm{cm} / \mathrm{s})\end{array}$ & $\begin{array}{c}\text { Velocity of } \\
\text { Polyaniline-Silica } \\
(\mathrm{cm} / \mathrm{s})\end{array}$ \\
\hline 1,000 & Non-movement & Non-movement \\
\hline 3,000 & Non-movement & Non-movement \\
\hline 5,000 & Non-movement & Non-movement \\
\hline 6,000 & 62.3 & 35.7 \\
\hline 7,000 & 41.5 & 47.2 \\
\hline
\end{tabular}

Table 4 Comparison of the surface electrical resistance of polymer-silica nanocomposite photo sheets and conventional photo sheet

\begin{tabular}{c|c}
\hline & $\begin{array}{c}\text { Surface electrical } \\
\text { resistance }(\Omega / \square)\end{array}$ \\
\hline Conventional photo sheet & $7.9 \times 10^{13}$ \\
\hline Coated $\mathrm{PPy}-\mathrm{SiO}_{2}$ sheet & $3.0 \times 10^{7}$ \\
\hline Coated $\mathrm{PANi}-\mathrm{SiO}_{2}$ sheet & $1.9 \times 10^{7}$ \\
\hline
\end{tabular}

ing polymer-silica nanocomposite particles size and cell gap size to the typical display elements size (around 10 $\mu \mathrm{m}$ ) and typical cell gap size (around $100 \mu \mathrm{m}$ ) for electrophoretic display, the minimum required applying electric voltage is $60 \mathrm{~V}$, the theoretical response time can be calculated to $62.3 \mathrm{~cm} / \mathrm{s}$ which is a sufficient speed for use in electrophoretic displays.

3.5 Electrical conductivity of a polypyrrole-silica nanocomposite paper sheet and a polyaniline-silica nanocomposite paper sheet

A summary of the experimental date of the electrical conductivities of polypyrrole-silica nanocomposite paper sheet and polyaniline-silica nanocomposite paper sheet are shown in Table 4. The paper sheet which are coated with polymer-silica nanocomposite particles have higher conductivity compared to a conventional paper. We confirmed that there is electrical conductivity on polymer-silica nanocomposite particles.

\section{Conclusions}

The polypyrrole-silica nanocomposite particles and polyaniline-silica nanocomposite particles represent a potentially useful processable form of polypyrrole and polyaniline, a normally intractable conducting polymer. The polymer-silica nanocomposite particles can be utilized as display electric for electrophoretic display due to their high colloid stabilities. The coated polymer-silica nanocomposite particles papers can be utilized as conductive paper due to their electrical conductivities.

\section{Acknowledgments}

We would like to express our sincere gratitude to Mr. Miyamoto of the Technical Service Coordination Office of Tokai University for their kind assistance with the TEM and SEM studies on the polypyrrole-silica nanocomposite particles and polyaniline--silica nanocomposite particles.

\section{References}

1) Y. Lee, Y. Wu and B. S. Ong: Facile synthesis of silver nanoparticles useful for fabrication of high-conductivity elements for printed electronics, J. Am. Chem. Soc., 127, pp. 3266-3267 (2005)

2) D. Kim and J. Moon : Highly conductive Ink Jet 
Printed Films of Nanosilver Particles for Printable Electronics, Electrochemical and Solid-State Letters, 8, pp. J 30-J 33 (2005)

3) Y. Lee, J. R. Choi, K. J. Lee, N. E. Stott and D. Kim : Large-scale synthesis of copper nanoparticles by chemically controlled reduction for application of inkjet-printed electronics, Nanotechnology, 19, 415604 (2008)

4) T. A. Skotheim and John R. Ryenolds : Handbook of Conducting Polymers Third Edition, CONJUGATED POLYMERS PROCESSING AND APPLICATIONS, CRC press Taylor \& Francis Group, pp. 8-14 8-17
(2007)

5) T. Sugiura, T. Syoji and S. Maeda : Preparation, Characterization, and Application of Organic Conducting Polypyrrole-Silica Nanocomposite Inks, Journal of the Imaging Society of Japan, 53, pp. 3-8（2014）

6) N. C. Billingham and P. D. Calvert, Advance in Polymer Science 90, Springer Verlag, Berlin, p. 73 (1989)

7) http://www.nissanchem.co.jp/products/materials/ snow_tex.html, (accessed 2013-04-16)

8) A. Kitahara, Kaimen koroido kagaku no kiso, Kodansha, pp. 103-128（1987）

(Manuscript accepted 9 September, 2015) 